\title{
Optimized In-focus Boersch Phase Contrast in a Dedicated TEM
}

\author{
B. Barton, ${ }^{*}$ K. Schultheiß,** M. Matijevic, ${ }^{* * *}$, D. Gerthsen** and W. Kühlbrandt* \\ * Dept. of Structural Biology, Max Planck Institute of Biophysics, Max-von-Laue-Str. 3, D-60438 \\ Frankfurt am Main \\ ** Lab. für Elektronenmikroskopie, Karlsruher Institut für Technologie (KIT), D-76128 Karlsruhe \\ *** Carl Zeiss NTS, Carl-Zeiss-Str. 56, D-73447 Oberkochen
}

Although recent technical developments have improved structure determination of frozen-hydrated biological objects by TEM, the fundamental problem of weak image contrast persists. In singleparticle cryo-EM or electron cryo-tomography, present technology does not provide sufficient signal-to-noise ratio (SNR) for low spatial frequencies $(>10 \mathrm{~nm})$, such as the outlines of soluble membrane protein complexes, simultaneously with high-resolution detail $(<1 \mathrm{~nm})$. As a result, structural analysis by cryo-TEM is mostly limited to particles of molecular weights above $300 \mathrm{kDa}$.

The problem of weak phase contrast can be overcome by a physical phase plate positioned in an objective lens diffraction plane. In analogy to quarter wave plates in light microscopy, the EM phase plate is used to change the relative phase of scattered and unscattered parts of the object exit wave by $90^{\circ}$, which results in an optimal phase contrast transfer function $p C T F_{\text {optimal }}=\cos \gamma \quad(\gamma$ : wave aberration). Phase plates of the Zernike type, consisting of thin amorphous films, have been successfully applied in a number of recent cryo TEM studies [1]. However, they have the disadvantage of coherence loss by electron scattering in the film, which limits the achievable resolution. Another technical implementation is the Boersch phase plate (Fig. 1), a micrometer-sized electrostatic einzel lens shifting the phase of the unscattered wave, thereby avoiding electron-matter interaction [2,3]. While such a device may already be used in materials science [4], its application to biology is more challenging because low spatial frequencies $k_{c u t-o n}<d / 2 \lambda f$ are obstructed by a lens of diameter $d$ (Table $1 ; \lambda$ : wavelength, $f$ : objective focal length).

We solved the problem of low-frequency obstruction by positioning a Boersch phase plate in a 5-fold magnified diffraction plane of a corrected $200 \mathrm{kV}$ TEM dedicated to low-dose cryo imaging. The prototype Phase Contrast Aberration Corrected EM (PACEM) has an effective focal length of 15 $\mathrm{mm}$ [5], which decreases the cut-on frequency to $d / 10 \lambda f$ (Table 1). The $\mathrm{C}_{\text {s }}$ corrector, monochromator and energy filter are used to compensate the increase in lens aberrations. In the PACEM, biological macromolecules can be imaged without significant loss of information (Fig. 1). We show that the effective $p C T F$ is phase-shifted by $90^{\circ}$ when applying a voltage of $\sim 500 \mathrm{mV}$ to the central electrode of the phase plate. This leads to an optimized transfer and SNR for object structure factors in images recorded close to focus (Fig. 2).

In future, optimized in-focus phase contrast will be applied to single particle structural analysis of ice-embedded macromolecules in the 50-300 kDa range, and for electron cryo-tomography.

References

[1] R. Danev and K. Nagayama, Journal of Structural Biology 161 (2008), 211-218

[2] E. Majorovits, B. Barton et al., Ultramicroscopy 107 (2007), 213-226

[3] R. Cambie et al., Ultramicroscopy 107 (2007), 329-339.

[4] Alloyeau et al., Ultramicroscopy 107 (2009), doi:10.1016/j.ultramic.2009.11.016.

[5] E. Majorovits et al., Microscopy and Microanalysis (2010). 
TABLE 1. Cut-on frequencies $k_{c u t-o n}$ for some TEMs when equipped with Boersch phase plates of different electrode lens outer diameters $d$.

\begin{tabular}{lccc}
\hline Microscope & Electron energy / focal length & $k_{\text {cut-on }}$ with $d=2.5 \mu \mathrm{m}$ & $(5.0 \mu \mathrm{m})$ \\
\hline FEI Spirit & $120 \mathrm{keV} / 2.8 \mathrm{~mm}$ & $1 / 7.5 \mathrm{~nm}$ & $(1 / 3.8 \mathrm{~nm})$ \\
JEOL FSC 3200 & $300 \mathrm{keV} / 4.4 \mathrm{~mm}$ & $1 / 6.9 \mathrm{~nm}$ & $(1 / 3.5 \mathrm{~nm})$ \\
PACEM & $200 \mathrm{keV} / 15 \mathrm{~mm}$ & $1 / 30 \mathrm{~nm}$ & $(1 / 15 \mathrm{~nm})$ \\
\hline
\end{tabular}
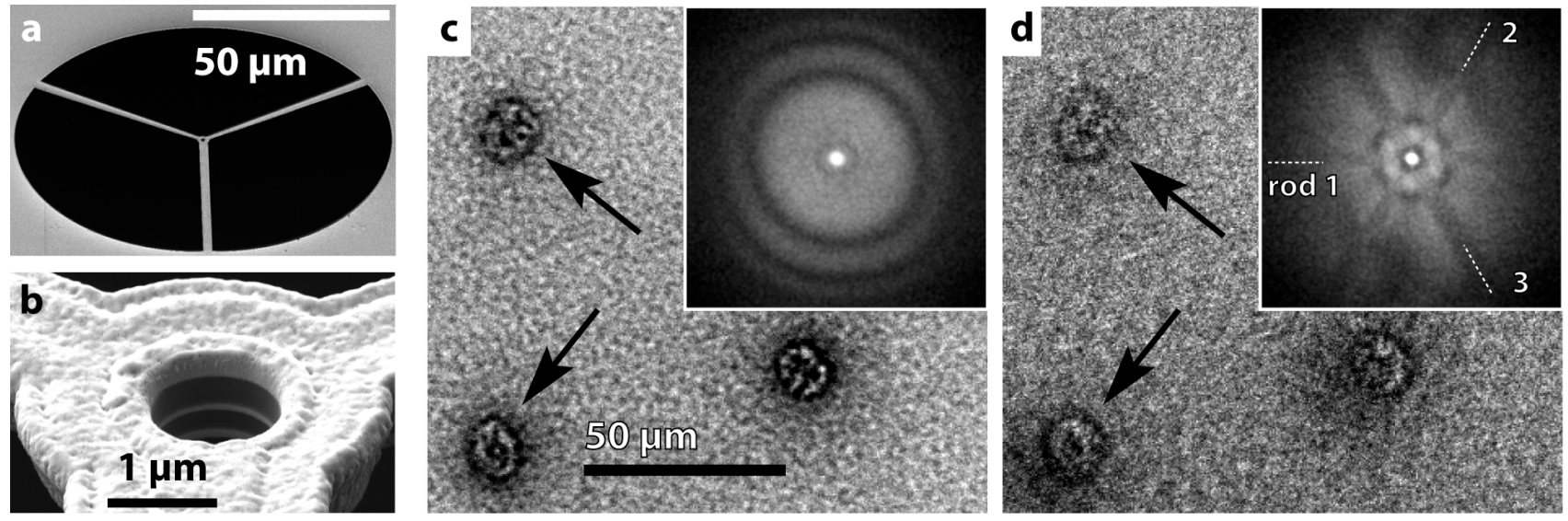

FIG. 1. (a) SEM image of the Boersch phase plate. (b) Close-up view of the einzel lens electrode with the central and shielding Au layers (bright). (c) Underfocused PACEM image of negatively stained Fatty Acid Synthase particles taken without phase plate. (d) Image of the same object, recorded close to focus with the phase plate electrode centered around the zero beam in the diffraction plane. The outline of the particles with $\varnothing \sim 10 \mathrm{~nm}$ can be clearly identified, proving that the 5-fold reduction of the cut-on frequency permits transfer of low spatial frequencies (see Table 1). Inset: power spectra with outline of the 3-fold support rod geometry; Nyquist frequency $\sim 0.46 \mathrm{~nm}^{-1}$.
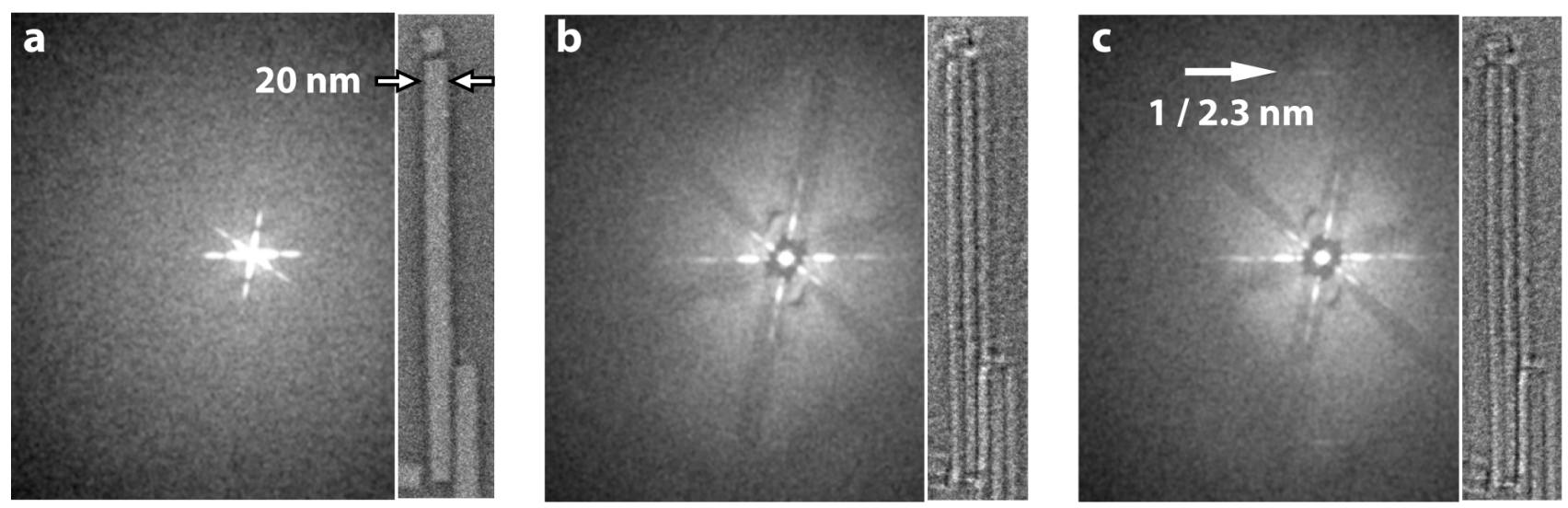

FIG. 2. Power spectra (left) and images (right) of a negatively stained TMV particle taken close to focus with the PACEM at $52 \mathrm{kx}$ primary magnification. (a) Without phase plate, the contrast is almost negligible. (b) Same imaging conditions as (a), with the Boersch phase plate centered on the optical axis and the central electrode grounded. Some phase contrast transfer can be observed (blackon-white contrast of support rod areas), most likely generated by phase shifts due to minor charging around the einzel lens. However, the SNR for the $2.3 \mathrm{~nm}$ TMV layer lines is low. (c) Same conditions as (b), with the central electrode at a potential of $+500 \mathrm{mV}$ relative to the TEM column. The SNR of the layer lines is now significantly improved. 